\title{
A Framework for the Metamodeling of Multi- variant Systems and Reactive Simulation Model Generation and Execution
}

\author{
Thorsten Pawletta ${ }^{1 *}$, Artur Schmidt ${ }^{1}$, Umut Durak ${ }^{2}$, Bernard P. Zeigler ${ }^{3}$ \\ ${ }^{1}$ Wismar University of Applied Sciences, Research Group Computational Engineering and Automation (CEA), \\ Philipp-Müller-Str. 14, D-23966 Wismar, Germany, *thorsten.pawletta@hs-wismar.de \\ ${ }^{2}$ German Aerospace Center (DLR), Institute of Fligth Systems, Lilienthalplatz 7, 38108 Braunschweig, Germany \\ ${ }^{3}$ RTSync Corp. 4425 E Agave Rd. Suite 106 Phoenix, AZ 85044, USA
}

SNE 28(1), 2018, 11 - 18, DOI: 10.11128/sne.28.tn.10402

Received: May 9, 2017 (Selected ASIM STS 2016 Postconf. Publ.), Accepted: December 15, 2017

SNE - Simulation Notes Europe, ARGESIM Publisher Vienna, ISSN Print 2305-9974, Online 2306-0271, www.sne-journal.org

Abstract. The simulation-based study of Cyber-Physical Systems or complex production systems leads often to a vast number of system variants. Each system variant is characterized by a particular model structure and parameter settings, although system variants may also share common parts. There are two main approaches for modeling such a set of system variants. On the one hand, all variants are mapped in a big model with variation points and on the other hand variants are specified on a higher level of abstraction using a metamodel that is processed with appropriate transformation methods. This paper proposes an approach for modeling system variants using the System Entity Structure (SES) Ontology. It introduces new concepts and advances the SES by a procedural knowledge specification. Moreover, it proposes a software infrastructure for the automated and reactive generation and execution of simulation models based on a SES in combination with a model base. Finally, it refers to a prototype implementation within MATLAB/Simulink and forward-looking within Python.

\section{Introduction}

The study of multifaceted end user requirements of Cyber-Physical Systems or of multi-variant production systems leads to a vast number of system variants. Both problem types can be considered as a variability problem.
Variability has been defined as the ability of a system or an artefact to be configured, customized or extended for employment in a particular context [1]. In software engineering Software Product Lines (SPL) are widely employed for developing systems that are characterized by a high degree of variability. SPL define variation points where different variants of products can be derived for varying requirements [2]. Variability management has also been introduced as a challenge to be tackled for model-based testing architectures [3], for model-based concept development tools [4] and for studying multi-variant production systems [5] or reactive robot controls [6]. In this context the problem of reactiveness is also discussed $[5,6,7]$. We will consider reactiveness as the generation and execution of a new system variant depending on current and previous results.

Variability mechanisms shall be defined at particular levels of abstraction, ranging from metamodeling to implementation of the source code. Using a metamodel for variability modeling requires appropriate model transformation methods for mapping to the execution level. This is a particular challenge, because such methods are not supported by the established modeling and simulation environments used in the engineering or production system domain. Another approach is the combination with software tools for requirement or variant management [8] or with domain oriented tools [4]. However, in this case often different kinds of models have to be maintained and kept consistent.

For these are reasons, in engineering and production system applications variability is still often encoded within the executable system models. However, these models are often hard to manage. 
Therefore, specific modularization and configuration methods have been developed to tackle the complexity. From the simulation theory the approach of dynamic structure or variable structure systems $[9,10]$ is known. On the other side, rather pragmatic solutions have been developed, such as for the MATLAB/Simulink environment in $[11,12]$.

For the modeling and simulation of modular, hierarchical systems, Zeigler introduced the System Entity Structure (SES) for specifying a set of system configurations, called a family of systems. The SES approach has evolved steadily to an ontology for model and data engineering $[13,14]$. In combination with a model base (MB), organizing a set of configurable basic models, the SES approach has been advanced to a modeling and simulation framework (SES/MB) [15]. In this paper, a reworked version of [16], we extend the SES ontology by adding new features. In addition, we advance the SES/MB framework to an infrastructure for reactive model generation and simulation execution and we refer to a prototype implementation. Using an exemplified multi-variant engineering problem, a concrete SES model, which is a metamodel, is developed. Based on the example, fundamental elements and axioms of the baseline SES ontology are briefly summarized. Next, some extensions to the SES ontology are discussed. The main new features are SESVariables and SESFunctions, which expand the SES ontology by procedural knowledge elements. After that, the selection of a concrete system variant from an SES metamodel is considered and the whole procedure for generating an executable simulation model is depicted under the aspect of reactiveness.

\section{Multi-variant Engineering Example}

The example is an extension of an application that has been introduced by The MathWorks in [12] to demonstrate features for variant modeling within MATLAB/Simulink. We use that example to make our approach comparable with The MathWorks solution for experienced users. The substantial problem statement is illustrated in Figure 1a. Different controller (ctrl) designs, based on a linear (lc) or a nonlinear (nc) control structure, should be investigated using different signal sources from a signal generator (sg).

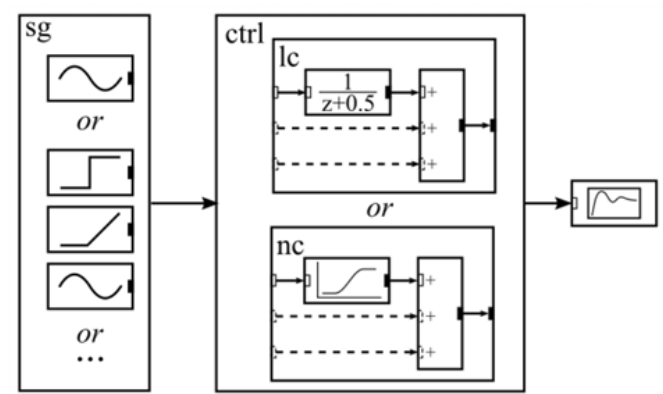

(a)

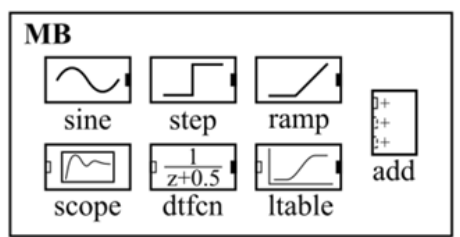

(b)

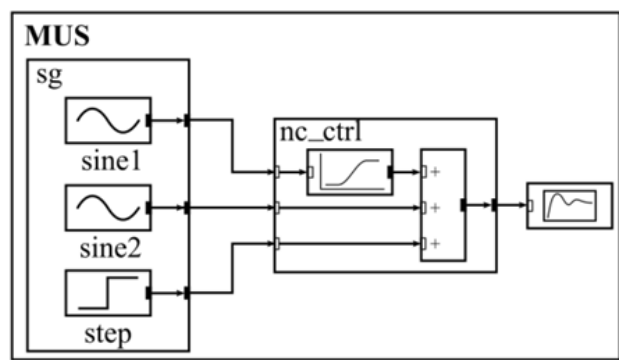

(c)

Figure 1: (a) Overall engineering problem with a set of system variants; (b) Blocks from the Simulink blockset representing the MB; (c) Model structure of a concrete system variant.

In addition to the control structure, the signal types $\{$ sine $\mid$ ramp $\mid$ step $\}$ and the number of signal sources $\{1 \ldots 3\}$ may vary. Figure 1a shows the two control approaches (lc_ctrl | nc_ctrl) as alternative submodels of model ctrl. Due to the varying number of possible input signals, both approaches lead to three different internal model structures. The minimal internal structure of a ctrl model with one input signal is illustrated with full lines. The extension for two or three input signals is pictured with dashed lines. In the same manner, the internal structure of the system generator (sg) depends on the number and type of included signal sources. Overall, the exemplary problem comprises $\left(3^{1}+3^{2}+3^{3}\right) * 2$ various system structures. All possible system structures can be aggregated using 7 basic systems. In this case, the basic systems are blocks from the Simulink blockset, which represent a model base (MB), as shown in Figure 1b. 
Figure 1c illustrates as an example the model structure of a specific system variant, which we call a model under study (MUS). In this case, the MUS consists of an nc_ctrl model, which is influenced by a sg model with three signal sources. Two sources are of type sine and one of type step. For simplification purposes, a separation between MUS and experimental frame (EF) according to [15] is not considered.

\section{Metamodel-Based Variant Modeling}

This section describes the specification of the exemplary problem to demonstrate multi-variant modeling using an SES. The specification is based on the baseline SES definitions in [13], but it uses some modifications based on former works in [17] and introduces some new concepts, such as the SESFunctions.

\subsection{SES fundamentals and SES variables}

The SES ontology supports the description of a family of systems regarding their elements and the relations between them. It is axiomatically defined and can be represented as a directed labeled tree. Nodes are divided into two types, entity and descriptive nodes, which can define specific attributes. Entity nodes describe system elements and the system itself (root node). The leaf nodes are always entity nodes, whose attributes define a link to a basic model in the MB (attribute $m b$ ) and possible parameter settings for the referenced basic model. Descriptive nodes express relationships between entities and are subdivided into: aspect, specialization and multi-aspect nodes.

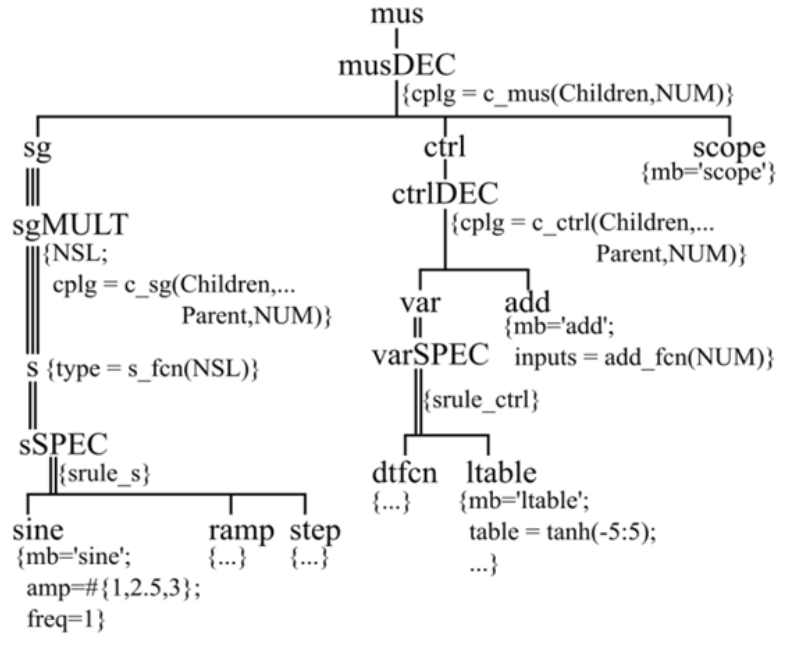

Figure 2: SES tree for the example in Figure 1a
The SES axioms will be considered subsequently, as necessary for the example. Figure 2 illustrates an SES tree that maps the problem described in Section 2. In the tree descriptive nodes are marked with name suffixes: (i) DEC for aspect, (ii) SPEC for specialization and (iii) MULT for multi-aspect. At this point the SES axiom alternating mode for entity and descriptive nodes should be noted.

Before describing the SES tree in detail, the new concept of SESVariables as the input interface of an SES is explained. This new feature was introduced to support the integration of an SES metamodel, referring to the metamodel definition in [18], in the later suggested infrastructure. In the infrastructure the selection of a particular system variant depends on the current settings of SESVariables. The selection procedure itself is described in the next section. SESVariables have a global scope and are written in uppercase letters in the tree. Two SESVariables in the tree in Figure 2 are defined as input arguments and a third one as an auxiliary variable. They have the following definitions:

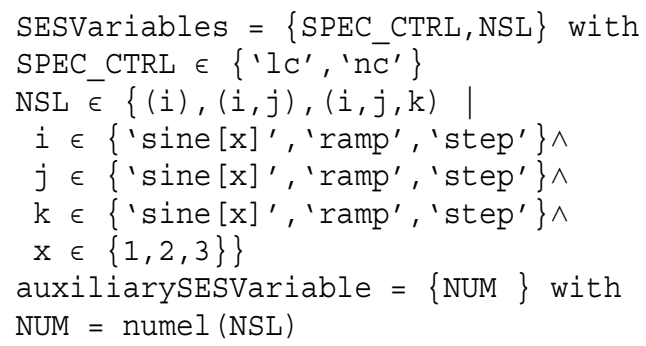

According to the exemplary problem (see Fig. 1), the variable SPEC_CTRL encodes the desired control structure and the variable $N S L$ specifies a list with the signal sources to be selected. The index value $x$ allows the encoding of different parameter selections for a sine signal. The auxiliary variable NUM calculates the current number of elements (numel) in NSL. An example for an admissible value assignment to SESVariables is given as follows.

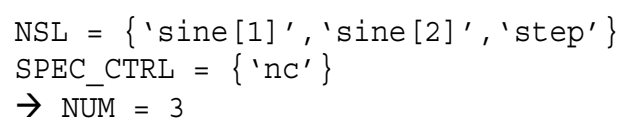

\subsection{Decomposition of systems with variable coupling relations}

The system itself (mus) is represented in the SES tree with the root node. The subsequent aspect musDEC and vertical lines define a decomposition of mus (parent) in the entities $s g, c t r l$ and scope (children). 
The aspect attribute $\{c p l g=\ldots\}$ defines the coupling relations of mus. Model couplings can be divided into internal couplings (IC) between children, and external input as well as external output couplings (EIC, EOC) between the parent and its children. However, a coupling relationship always has the following structure:

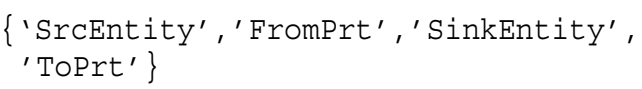

In the example some ICs of entity mus depend on the number of signal sources defined by $s g$ (see Fig. 1). To express such dynamics with minimal effort and to keep a lean SES tree, the concept of SESFunctions has been introduced. The SESFunctions are like ordinary functions. They extend the declarative specification defined by the baseline SES by procedural knowledge descriptions. SESFunctions are calculated during the processing of an SES, called pruning, and are described in the next section. This means that the terms:

cplg = c mus (Children, NUM)

cplg = c_ctrl (Children, Parent, NUM)

represent ordinary function calls that return the coupling relations, which depend on the current settings of the input arguments. The variables Children and Parent are implicit attributes of each tree node, which save the names of the successor (left-to-right) and predecessor nodes. Hence, the set of variable couplings of entity $m u s$, derived from the overall problem illustrated in Figure 1, can be defined using the following SESFunction (in MATLAB syntax):

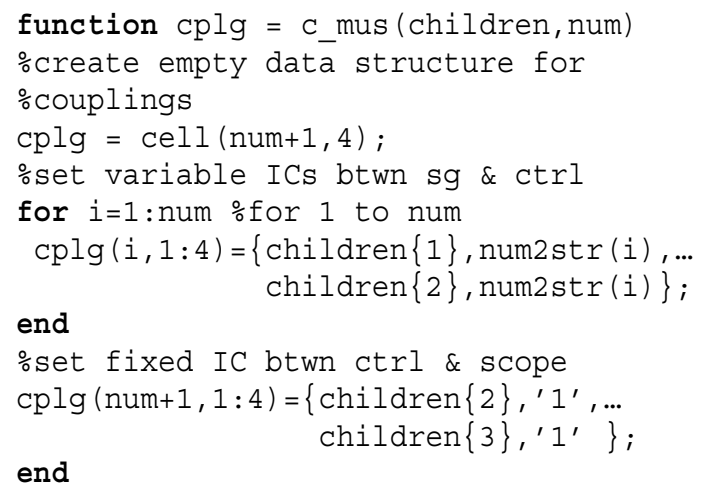

The children sg and ctrl of mus are composed entities, while scope is an atomic entity. Leaf node scope maps a basic system in the SES and defines with its attribute $m b=$ 'scope' a corresponding link to the MB. The decomposition of entity ctrl in the entities var and $a d d$ is specified by its successor node $c t r l D E C$. In both control approaches, the linear and nonlinear (see Fig. 1), the coupling relations of $c t r l$ depend on the number of external inputs, which again depend on the current number of signal sources. Thus, the coupling relations at ctrlDEC are specified by an SESFunction analogous to node $m u s D E C$.

\subsection{Variable system attributes and the specialization of systems}

Leaf node add represents a basic model, such as node scope. In contrast to scope, it defines a variable attribute for parameter settings, using the SESFunction call input $s=a d d \_f c n(N U M)$. As illustrated in Figure 1, the configuration of add depends on the number of inputs. This problem is specified with the following SESFunction (MATLAB syntax):

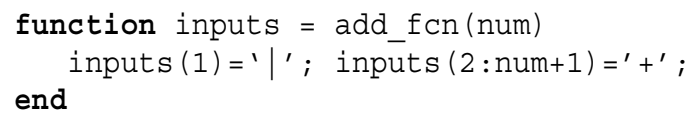

The characteristic of entity node var is specified by the succeeding specialization node varSPEC, marked with double-line edges. A specialization describes an is- $a$ relation concerning the succeeding nodes; in this case, entity var can be $d t f c n$ or ltable. While processing an SES, the selection is controlled by evaluating selection rules that are specified as node attribute. In this case the following rule is defined.

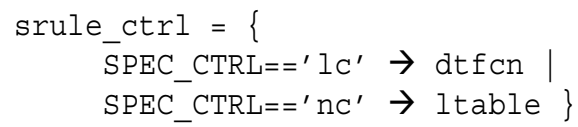

For specializations the specific SES axiom inheritance is defined. Its effects will be explained in the next subsection. The leaf nodes $d t f c n$ and ltable represent once again basic models. The node ltable shows a further example for a variable attribute definition.

\subsection{Variable decomposition of systems}

According to the problem description in Section 2, the node $s g$, following the aspect $m u s D E C$, represents a system entity composed of a variable number of signal sources of various types. Referring to the baseline SES definition, such selection and composition has to be specified using a combination of aspect or multi-aspect and specialization nodes, possibly supplemented by selection constraints. However, this approach quickly leads to a confusing SES tree. In the following, an approach for keeping a lean SES tree will be described.

In former work [17] regarding concepts of SES, a first idea for solving this specific problem was discussed under the constraint of relaxing the SES strict hierarchy axiom. 
Based on this idea, we will suggest a complete solution without the violation of the strict hierarchy axiom. In Figure 2 the entity $s g$ is characterized by the succeeding multi-aspect $s g M U L T$ with triple-line edges. According to the baseline SES definition, a multi-aspect is a special case of an aspect in which the succeeding entities are homogeneous in nature. Thus, it has only one succeeding entity node and defines the number of replications of this node as an attribute. Accordingly, the node $s g M U L T$ has one succeeding entity node named $s$. However, the node attribute definitions of the multi-aspect $s g M U L T$ and the succeeding entity $s$ are more complex referring to the baseline SES definition. Node $s g M U L T$ specifies in the SESVariable NSL a list of types for replication. The number of replications is implicitly specified by the number of list elements. Remember the example

$$
\text { NSL }=\{\text { 'sine }[1] ', \text { 'sine [2]', 'step'\} }
$$

stated at the end of Subsection 3.1. Furthermore, sgMULT defines variable coupling relations using the SESFunction call $\mathrm{cplg}=\mathrm{C}_{-} \mathrm{sg}(. .$.$) , analogous to the$ aspect nodes $m u s D E C$ and $c t r l D E C$.

The entity $s$ specifies an attribute type. The concrete value of this attribute is determined by calling the SESFunction $s \in f C n$ (NSL) when processing the SES. The SESFunction $s_{-} f \mathrm{fn}$ defines a simple iterator.

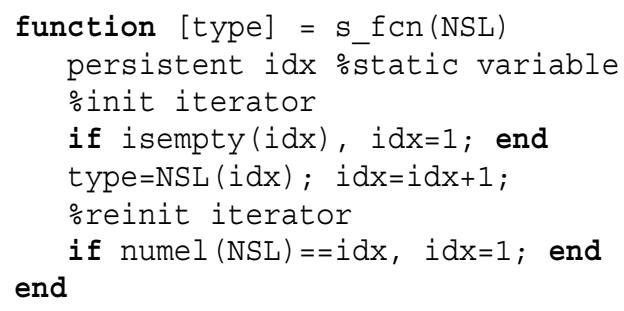

Thus, for each replication of entity $s$ an individual value assignment is made, such as in our supposed case type $=$ 'sine [1]', type ='sine [2]' and type='step', when processing the SES. Based on the setting of attribute type, replications of entity $s$ can be specialized using a succeeding specialization node. This is specified in the SES tree with the node $S S P E C$, which defines the various signal sources as succeeding entities and the following selection rule as its attribute.

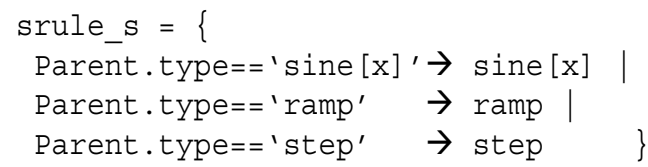

This means that the selection at $S S P E C$ depends on the value assignment to attribute type at the parent node of $S S P E C$. Details of this subject will be discussed in the next section (see Fig. 4).

The leaf node entities sine, ramp and step once again represent basic systems, which specify a link to the MB and parameter configurations. The attribute $a m p=\#\{1,2.5,3\}$ of entity sine defines an ordered multiset for different parameter configurations. Therefore, specifications referring to a sine signal source are extended by the index $x$ to choose an element from the multiset amp.

\section{Selecting a Distinct System Variant}

An SES, such as in Figure 2, codes a set of system variants and is a metamodel referring to the definition in [18]. For simulation studies a single or several distinct system variants must be derived from the SES metamodel. The selection of a particular model structure, including parameter settings for basic models, depends on the current settings of SESVariables and the selection itself is performed by graph pruning. The result of pruning is a decision-free tree, called Pruned Entity Structure (PES), which contains all of the necessary knowledge for building a distinct simulation model using basic models from the MB. Figure 3 shows one PES derived from the SES in Figure 2 using the subsequent value assignments to the SESVariables.

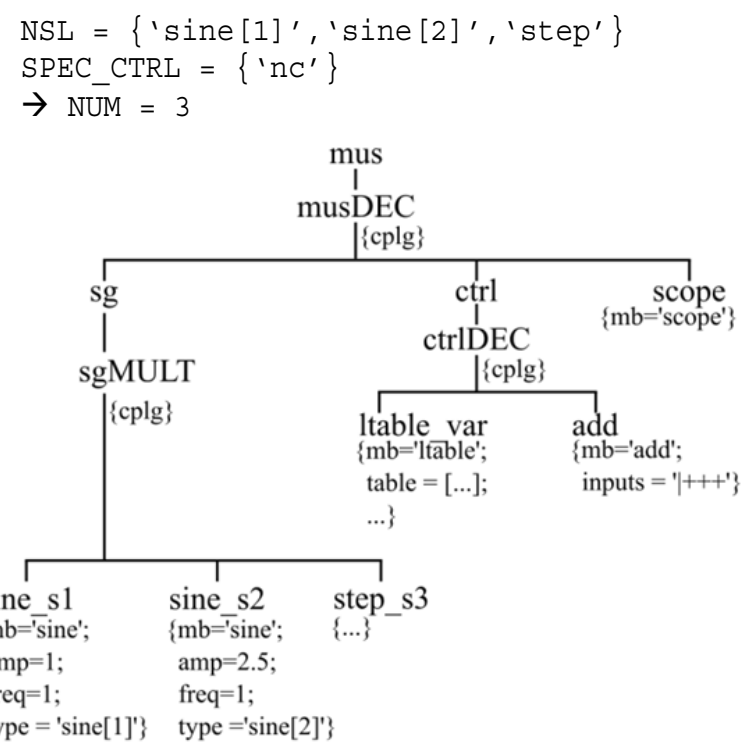

Figure 3: PES derived from SES in Figure 2 
The PES in Figure 3 codes a system structure analogous to the MUS in Figure 1c. Subsequently, we will describe the pruning operation in detail. Starting at the root node of the SES in Figure 2, the first decision operation occurs at aspect musDEC. The SESFunction called $c p l g=c \_$mus (Children,3) is executed to determine the coupling relations. The result is:

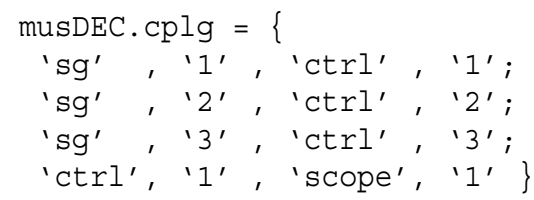

The next decision point is at multi-aspect $\operatorname{sg} M U L T$. According to the number of elements in SESVariable $N S L$, the entity $s$, including its following sub-tree, has to be replicated three times. During this operation replicas of $s$ are renamed to comply with the valid brothers axiom. Moreover, any replica is assigned an exact value to its attribute type by executing the iterator SESFunction $s_{-} f c n$ with the input argument $N S L=\{$ 'sine [1]', 'sine [2]', 'step' $\}$. The results of this operation are the replicated and renamed entities $s 1, s 2$, $s 3$ with their identical sub-trees but an individual value assignment to their attribute type, as illustrated in Figure 4. Now, for each entity $s_{i}$ the replicated sub-tree is evaluated. This means that the selection rule srule_s is evaluated for each node $S S P E C$. In our case, it delivers the following selection sine [1], sine[2] and step. Remember, the indices of sine denote the parameter selection for the multiset of attribute amp .

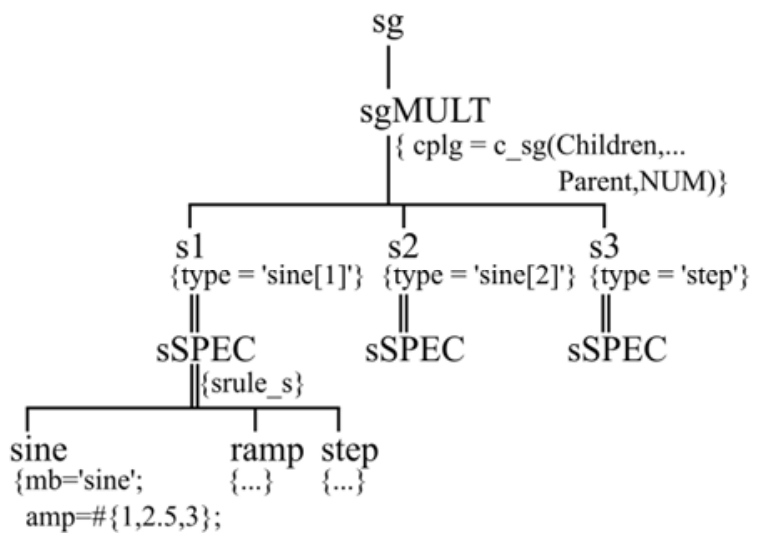

Figure 4: Part of 'intermediate PES' (sub-tree of sg) during pruning of multi-aspect sgMULT

Next, the parent and child entity of each specialization relation is combined according to the inheritance axiom.
In this case, only the entity names and attributes have to be combined, e.g. sine_sl $\{m b=$ 'sine';...; type $=$ 'sine [1]' $\}$. Finally, the current coupling relations, specified at node $s g M U L T$, are determined by executing the SESFunction call $c p l g=c_{-} s g$ (Children, Parent, $N U M)$. The result is:

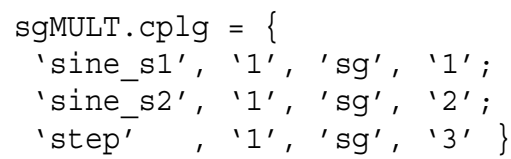

The sub-tree of entity ctrl in Figure 2 is resolved in a similar manner during pruning. The resulting coupling relations for entity $c t r l D E C$ are the following:

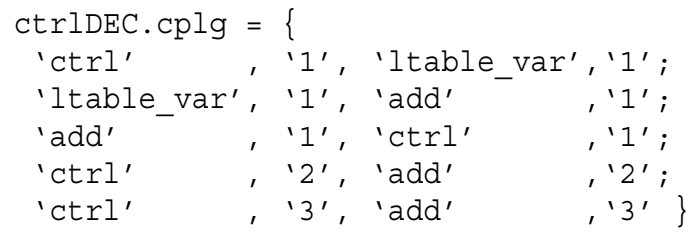

As mentioned in the beginning, the PES contains all of the necessary knowledge for building a simulation model using basic models from the MB. Sometimes, the PES contains unnecessary attributes due to the pruning operation, such as type in the entities sine_sl and si$n e \_s 2$, which can be neglected when building the simulation model. Moreover, referring to [15], the PES can be flattened by restructuring. Then, in our case the inner nodes sg, sgMULT, ctrl, ctrlDEC are resolved and all coupling relations are restructured in the $c p l g$ attribute of aspect musDEC.

\section{Software Infrastructure and Prototype Implementation}

Figure 5 shows the proposed infrastructure for multivariant modeling and reactive model generation and execution. The core element is the SES/MB framework according to [15], which is extended by an input and output interface using the introduced SESVariables. This part of the infrastructure maps the functionality as described in the previous sections: (i) basic models are organized in an MB; (ii) the set of system variants is specified in an SES; (iii) the selection of a particular system variant depends on the current settings of SESVariables, it is performed by the pruning operation and its result is a decision-free tree structure, called PES. 
Then, an executable simulation model (EM) can be generated based on the PES and basic models from the MB using an appropriate translation method. The composition of EM as tuple (MUSi, EFj) means that it consists of a Model Under Study (MUS) and a corresponding Experimental Frame (EF), according to the theory in $[15,19]$. The indices $\mathrm{i}$ and $\mathrm{j}$ are markers for a certain system configuration. An EM is transmitted to the Execution Unit (EU). The EU performs three major tasks: (i) linking an EM with a simulation engine; (ii) executing a simulation run; and (iii) collecting the results.

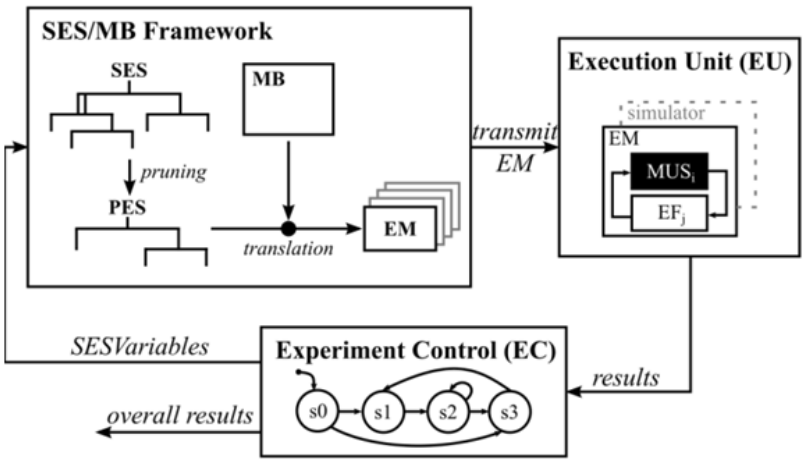

Figure 5: Infrastructure for multi-variant modeling and reactive model generation and execution.

Once the execution phase is complete, the results are sent to the Experiment Control (EC). The EC manages the order of EM generations and executions. Based on an application-dependent algorithm, the EC computes new settings for SESVariables as current input for the SES/MB framework to start the next cycle. Additionally, the EC collects all intermediate results from the EU. By means of the feedback loop, structural changes of a variable structure system or experiments with several system configurations can be executed in a reactive manner. Finally, the EC provides the overall results to the user or another software component.

For the investigation of multiple system configurations, such as in our introduced engineering example, it can be useful to generate an EM suite, as illustrated in Figure 5, and to execute it in a sequential or distributed manner by the EU.

The proposed infrastructure has been implemented in the MATLAB/Simulink environment. Thus, a parallel or distributed execution of EMs by the EU is directly supported. Basic implementation aspects of the infrastructure within MATLAB/Simulink and its usage for solving a specific class of multi-variant problems are discussed in [20].
A core element of the infrastructure is the SES toolbox for MATLAB/Simulink, which has been developed by the Research Group CEA [21, 22]. The toolbox provides a graphical SES editor and several methods, such as: (i) merging to synthesize various SESs'; (ii) pruning for deriving a PES; (iii) flattening for the hierarchy reduction of a PES; (iv) validity checking of an SES and PES; and (v) translation scripts or templates to build EM for Simulink or MATLAB/DEVS [23]. Advanced engineering applications for deploying the SES toolbox for MATLAB/Simulink in the field of modelbased testing can be found in [20]. Moreover, a new prototype of the SES toolbox, implemented with Python and supporting an XML interface, is in development to open the way for investigating the approach in conjunction with other simulation environments.

\section{Conclusion}

Multi-variant modeling and reactive model generation and execution is an important requirement in systems and production engineering. This paper presented a metamodel-based approach using the SES ontology and introduced an appropriate infrastructure to solve this requirement. In addition to the baseline SES definition, the approach uses some new extensions which have been explained step by step based on an engineering example. The introduced concept of SESFunctions advances the declarative knowledge representation through a procedural knowledge specification. Particularly for the modeling of systems with a high degree of variability, the SESFunctions support maintaining a lean SES even for complex problems.

In a next step, this assumption has to be proven by applying the approach to more complex examples. The discussed infrastructure, implemented within MATLAB/Simulink, provides a basis for solving more complex engineering problems. Currently, it is used for developing the reactive and structure variable controls of interacting industrial robots and in the field of objective fidelity evaluation of flight and research simulators. Moreover, a new prototype of the SES toolbox, implemented with Python and supporting an XML interface, is in development to open the way for investigating the approach in conjunction with other simulation environments. 


\section{ACKNOWLEDGMENTS}

The authors, T. Pawletta and A. Schmidt, gratefully acknowledge the grant from the German Science Foundation DFG (PA 631/2).

\section{References}

[1] Capilla R, Bosch J, Kang KC. Systems and Software Variability Management. Springer Pub., 2013.

[2] Polzer A, et al. Managing complexity and variability of a model-based embedded software product line. Innovations System Software Engineering. 2012, Vol. 8, 35-49.

[3] Sargardui G, Etxeberria L, Agirre JA. Variability management in testing architectures for embedded control systems. In: The 4th Int. Conf. on Advances in System Testing and Validation Lifecycle (VALID 2012). IARIA Press, 2012, 73-78.

[4] Krausz M, Zimmer M, Reuss HC. OverNight Testing The fully automated simulation environment for evaluation of car concepts. SNE - Simulation Notes Europe, 2014, Vol. 24(2), 87-94.

[5] Schmidt A, Pawletta T.: Ein Ontologie-basierter Modellierungs- und Simulationsansatz am Beispiel der ressourceneffizienten Planung spanender Prozessketten. In: Proc. 15. ASIM Fachtagung Simulation in Produktion und Logistik, Paderborn, HNI-Verlagsschriftenreihe Bd. 316, 2013, 481-490.

[6] Schwatinski T, Pawletta T, Pawletta S. Flexible Task Oriented Robot Controls Using the System Entity Structure and Model Base Approach. SNE - Simulation Notes Europe, 2012, Vol. 22(2), 107-114.

[7] Zander J. Model-based Testing of Real-Time Embedded Systems in the Automotive Domain. PhD Thesis, TU Berlin, 2008

[8] Beuche D. Modeling and building software product lines with pure::variants. In: Proc. $12^{\text {th }}$ Int. Software Product Line Conf., IEEE Comp. Soc., 2008, 358-358.

[9] Barros FJ. The dynamic structure discrete event system specification formalism. Transactions of the SCS International, 1996, No.1, 35-46.

[10] Pawletta T, Lampe B, Pawletta S, Drewelow W. A DEVS-based approach for modeling and simulation of hybrid variable structure systems. In: Modeling, Anlysis, and Design of Hybrid Systems, Lecture Notes in Control and Information Sciences 279, Springer Pub. , 2002, 107-129.
[11] Haber A. et al. First-class variability modeling in MATLAB/Simulink. In: Proc. of the 7th Int. Workshop on Variability Modelling of Software-intensive Systems. ACM Press, 2013, 11-18.

[12] The MathWorks. Variant management. http://de.mathworks.com/help/simulink/ug/variantmanagement.html, Date of reading 12-Nov. 2015.

[13] Zeigler BP, Hammonds PE. Modeling and SimulationBased Data Engineering. Academic Press, 2007.

[14] Zeigler BP, Sarjoughian HS. Guide to Modeling and Simulation of Systems of Systems. Springer Pub., 2012.

[15] Zeigler BP, Prähofer H, Kim TG. Theory of Modeling and Simulation. Academic Press, 2000.

[16] Pawletta T, Schmidt A, Zeigler BP, Durak U. Extended Variability Modeling Using System Entity Structure Ontology within MATLAB/Simulink. In: Proc. SprigSimANSS, 2016, Pasadena, USA, 62-69.

[17] Rozenblit JW, Zeigler BP. Representing and constructing system specifications using the system entity structure concepts. In: Proc. of the Winter Simulation Conf., 1993, 604-611.

[18] Pidcock W, Uschold M. What are the differences between a vocabulary, a taxonomy, a thesaurus, an ontology, and a meta-model? In: The Web Graph Database, http://infogrid.org/trac/wiki/ Reference/PidcockArticle, Date of reading 7-July 2016.

[19] Traoré M, Muzy A. Capturing the dual relationship between simulation models and their context. Simulation Modelling Practice and Theory, 2006, No. 2, 126-142.

[20] Schmidt A, Durak U, Pawletta T. Model-based testing methodology using system entity structures for MATLAB/Simulink models. Simulation: Transactions of SCS Int., Sage Pub., 2016, Vol. 92(8), 729-746.

[21] Pawletta T, Pascheka D, Schmidt A, Pawletta S. Ontology-assisted system modeling and simulation within MATLAB/Simulink. SNE - Simulation Notes Europe, 2014, Vol. 24(2), 59-68.

[22] Pawletta T, Schmidt A, Pascheka D. SES Toolbox for MATLAB/Simulink. https://www.mb. hswismar.de/cea/SES_Tbx/, Date of reading 12-Nov. 2015.

[23] Deatcu C, Schwatinski T, Pawletta T. DEVS Toolbox for MATLAB. https://www.mb.hs-wismar.de/cea/SES_Tbx/, Date of reading 12-Nov. 2015. 